\title{
25-Hydroxyvitamin D insufficiency is associated with cardiometabolic risk in Korean adolescents: the 2008-2009 Korea National Health and Nutrition Examination Survey (KNHANES)
}

\author{
Ga Eun Nam", Do Hoon Kim', Kyung Hwan Cho ${ }^{1, *}$, Yong Gyu Park², \\ Kyung Do Han ${ }^{2}$, Seon Mee Kim ${ }^{1}$, Seung Hwan Lee ${ }^{1}$, Byung Joon Ko ${ }^{1}$ and Min Ji Kim ${ }^{1}$ \\ 'Department of Family Medicine, Korea University Anam Hospital, Korea University College of Medicine, \\ 126-1, Anam-dong 5-ga, Seongbuk-gu, Seoul 136-705, Republic of Korea: ${ }^{2}$ Department of Biostatistics, \\ Catholic University of Korea College of Medicine, Seoul, Republic of Korea
}

Submitted 1 September 2011: Final revision received 13 September 2012: Accepted 25 September 2012: First published online 20 November 2012

\begin{abstract}
Objective: Vitamin D insufficiency is known to be related to cardiometabolic disorders; however, the associations among serum 25-hydroxyvitamin D (25(OH)D) concentration and metabolic syndrome and cardiometabolic risk factors in children and adolescents have not yet been clearly delineated. For this reason, we investigated the relationship among serum 25(OH)D concentration and metabolic syndrome and cardiometabolic risk factors among Korean adolescents.

Design: We performed a cross-sectional analysis and used hierarchical multivariate logistic regression analysis models to adjust for confounding variables.

Setting: We used the data gathered during the 2008-2009 Korea National Health and Nutrition Examination Survey (KNHANES).

Subjects: Our subjects included 1504 Korean adolescents aged 12-18 years who participated in the KNHANES.

Results: Vitamin D insufficiency, defined as $25(\mathrm{OH}) \mathrm{D}$ concentration $<50 \mathrm{nmol} / \mathrm{l}$, was found in $75.3 \%$ of Korean adolescents and was associated with an increased risk of the prevalence of metabolic syndrome. Waist circumference and BMI were the most closely correlated cardiometabolic components of metabolic syndrome according to serum $25(\mathrm{OH}) \mathrm{D}$ status, but no significant relationship was found between serum $25(\mathrm{OH}) \mathrm{D}$ concentration and insulin resistance or for the risks for high blood pressure, hyperglycaemia, reduced HDL-cholesterol or hypertriacylglycerolaemia, with or without adjustment for confounding variables. Conclusions: Low serum 25(OH)D concentration appears to be associated with several cardiometabolic risk factors and an increased risk of the prevalence of metabolic syndrome in Korean adolescents.
\end{abstract}

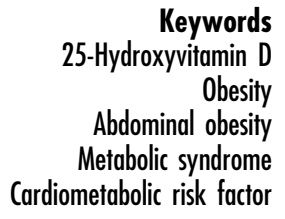

Vitamin D is an essential component for intestinal absorption of $\mathrm{Ca}$, playing an important role in bone growth and the homeostasis of minerals and $\mathrm{Ca}^{(1)}$. In laboratory and epidemiological studies, the actions of vitamin $\mathrm{D}$ have gained renewed attention since it was recently reported that multiple tissues and cells in the human body appear to express vitamin D receptors and $1 \alpha$-hydroxylase, which are necessary for converting vitamin $\mathrm{D}$ into its active form ${ }^{(2)}$. Some studies have demonstrated that serum 25-hydroxyvitamin D level $(25(\mathrm{OH}) \mathrm{D})$ not only indicates the status of bone health, but is relevant to several chronic diseases, including metabolic syndrome, cardiovascular, autoimmune and infectious diseases, and cancer ${ }^{(3,4)}$.
Despite new information on the physiological actions of vitamin $\mathrm{D}$, vitamin $\mathrm{D}$ deficiency in children and adults has been documented worldwide. Notably, children and young adults seem to be highly prone to vitamin D deficiency ${ }^{(2,5,6)}$. Concentrations of $25(\mathrm{OH}) \mathrm{D}$ are the most commonly used indicator of vitamin D status ${ }^{(7)}$. There is a lack of consensus regarding the optimal serum $25(\mathrm{OH}) \mathrm{D}$ concentration; however, $50 \mathrm{nmol} / 1(20 \mathrm{ng} / \mathrm{ml})$ remains a generally agreedupon cut-off between 'sufficiency' and 'insufficiency' of vitamin $\mathrm{D}$ in children and adolescents ${ }^{(8,9)}$.

It has been reported that serum $25(\mathrm{OH}) \mathrm{D}$ concentration is associated with metabolic syndrome and its components, including obesity, hypertension, hyperglycaemia and dyslipidaemia, although no consensus has been reached about 
this association either cross-sectionally or prospectively ${ }^{(10-12)}$. Further, several cross-sectional studies have found that vitamin $\mathrm{D}$ deficiency appears to be related to increased risk for $\mathrm{CVD}^{(1,13)}$. However, most of the aforementioned studies have been conducted in Western countries. In Asia, the relationship between vitamin D status and the risk of metabolic syndrome has been reported among middle-aged and elderly Chinese people ${ }^{(14)}$. There have been studies regarding the status of serum $25(\mathrm{OH}) \mathrm{D}$ in all age groups ${ }^{(15)}$, and the association between vitamin $\mathrm{D}$ and cardiometabolic risks has been made only in Korean adults ${ }^{(10,16)}$. No attempts have been made to investigate the association among serum $25(\mathrm{OH}) \mathrm{D}$ concentration and metabolic syndrome and cardiometabolic risk factors in children and adolescents in East Asia, including Korea.

To this end, the present study identified the status of serum $25(\mathrm{OH}) \mathrm{D}$ concentration among Korean adolescents who took part in the 2008-2009 Korea National Health and Nutrition Examination Survey (KNHANES) and investigated the relationships among serum 25(OH)D concentration and metabolic syndrome and cardiometabolic risk factors.

\section{Methods}

\section{Overview of survey and study participants}

The present study is based on data collected in 2008-2009 ${ }^{(17)}$, the second and third year of the fourth Korea National Health and Nutrition Examination Survey (KNHANES IV) conducted from 2007 to 2009. The KNHANES is a nationally representative survey conducted by the Division of Chronic Disease Surveillance under the Korea Centers for Disease Control and Prevention on an annual basis since 1998 to accurately determine national health and nutrition levels and for the purpose of gaining basic data.

The survey consists of a health interview survey, a nutrition survey, a health examination survey and data on demographic characteristics, diet and health, collected through personal interview. Physical examinations, blood sampling and urine sampling were carried out at a mobile examination centre. The sampling frame was based on the 2005 population and housing census in Korea. A stratified, multistage probability sampling design was used for the selection of household units that participated in the survey.

In the KNHANES IV, there were 264186 primary sampling units, each of which had sixty households. Two hundred units from the primary sampling units were randomly selected, and then twenty-three households from each unit were sampled using a systemic sampling method in the second and third years (2008-2009). Finally, 25250 individuals in 9200 households were sampled: 19386 participated in the health interviews and health examination surveys and 18038 in the nutrition surveys. For the purpose of the present analysis, adults aged 19 years and older and children younger than 12 years were excluded. There were 1670 eligible adolescents aged $12-18$ years who participated in the survey, which took place from February 2008 to December 2009. Eleven adolescents were excluded for congenital heart disease, and four were excluded for having been previously diagnosed with epilepsy. To ensure that $25(\mathrm{OH}) \mathrm{D}$ data had been measured in a single centre, seventy-nine adolescents who were examined in Seoul Clinical Laboratories were excluded. Seventy-two adolescents who had not fasted for at least $8 \mathrm{~h}$ prior to blood sampling were also excluded. Ultimately, data from 1504 adolescents (792 boys and 712 girls) were utilized. Since all participants in the present study were considered minors, their parents signed informed consent forms. The institutional review board of the Korea Centers for Disease Control and Prevention approved the study protocol.

\section{Description of demographic variables}

Demographic variables that were expected confounding factors in the present study include gender, age, monthly income, alcohol drinking and smoking status, use of multivitamin or mineral supplements, regular physical exercise, sleep duration, and daily energy and Ca intakes.

The study participants were divided into two groups, aged 12-15 years and 16-18 years. Monthly income levels based on Euros were subdivided into three groups: $<712 €$ (equivalent to 1 million wons (South Korean currency)), $712-2135 €$ and $>2135 €$ (equivalent to 3 million wons). Alcohol drinking was subdivided into two groups based on the frequency of drinking for the past year: less than once monthly and at least once monthly (once monthly or more than once monthly). Smoking was subdivided into smokers and non-smokers based on present smoking status. Based on the questionnaire responses on multivitamin or mineral supplements use, respondents were divided into two groups (yes or no). Regular physical exercisers were considered those who performed moderate exercise more than five times weekly for over $30 \mathrm{~min}$ per session, or engaged in vigorous exercise more than three times weekly for over 20 min per session. In terms of sleep duration, three groups were set based on the average number of daily sleeping hours: $<6 \mathrm{~h}, 6-8 \mathrm{~h}$ and $>8 \mathrm{~h}$. Regarding food intake, a $24 \mathrm{~h}$ recall method was used to determine the foods consumed on the previous day. Energy and $\mathrm{Ca}$ intakes were based on the food database developed for the KNHANES and the food composition tables published by the National Rural Living Science Institute under the Rural Development Administration.

\section{Antbropometric measurements}

Body weight and height were measured to the nearest $0 \cdot 1 \mathrm{~kg}$ and $0 \cdot 1 \mathrm{~cm}$, respectively, with the participants in light indoor clothing without shoes. BMI was calculated 
using the formula: weight $(\mathrm{kg}) /[\text { height }(\mathrm{m})]^{2}$. Waist circumference (WC) was measured at the narrowest point between the lower border of the rib cage and the iliac crest. To measure systolic blood pressure (SBP) and diastolic blood pressure (DBP), a standard mercury sphygmomanometer (Baumanometer; W.A. Baum Co., Inc., Copiague, NY, USA) was used on the right arm. SBP and DBP were measured twice at 5 min intervals, and the average values were used for the analysis. A determination of overweight or obesity in adolescents was based on the modified National Cholesterol Education ProgramAdult Treatment Panel III (NCEP-ATP III) criteria ${ }^{(18)}$ and gender-specific and age-specific examinations were performed using the 2007 Korean children and adolescent national growth chart ${ }^{(19)}$. According to these criteria, $\mathrm{BMI} \geq 25 \cdot 0 \mathrm{~kg} / \mathrm{m}^{2}$ or gender- and age-specific BMI $\geq 95$ th percentile was defined as obese, while $\mathrm{BMI} \geq 85$ th to $<95$ th percentile was diagnosed as overweight.

\section{Biochemical measurements}

To measure concentrations of serum fasting plasma glucose (FPG), total cholesterol (TC), TAG, HDL-cholesterol (HDL-C), white blood cell (WBC) count, insulin and serum 25(OH)D, a blood sample was collected from the antecubital vein of each participant after fasting for $>8 \mathrm{~h}$. Blood samples were appropriately processed, immediately refrigerated and transported in cold storage to the Central Testing Institute in Seoul, Korea. Blood samples were analysed within $24 \mathrm{~h}$ of transportation. Serum 25(OH)D levels were measured using a gamma counter (1470 Wizard; PerkinElmer, Wallac, Turku, Finland) by RIA using a 25-hydroxyvitamin D ${ }^{125}$ I RIA kit (DiaSorin, Stillwater, MN, USA). Levels of FPG, TC, TAG and HDL-C were measured with a Hitachi Automatic Analyzer 7600 (Hitachi, Tokyo, Japan) by enzymatic methods using commercially available kits (Daiichi, Tokyo, Japan). Insulin levels were determined using a gamma counter (1470 Wizard, Perkin Elmer) with an immunoradiometric assay using a Biosouce INS-IRMA kit (Biosource Europe SA, Nivelles, Belgium). WBC counts were measured using a Sysmex XE-2100D (Sysmex, Kobe, Japan) by laser flow cytometry. Insulin resistance (homeostasis model assessment of insulin resistance (HOMA-IR)) was calculated as fasting insulin $(\mu \mathrm{U} / \mathrm{ml}) \times$ fasting glucose $(\mathrm{mg} / \mathrm{dl}) / 405^{(20)}$.

\section{Description of metabolic syndrome}

Metabolic syndrome in adolescents was defined according to the modified NCEP-ATP III criteria ${ }^{(18)}$. According to these criteria, metabolic syndrome is diagnosed when an individual has three or more of the following components: (i) WC $\geq 90$ th percentile for age and sex; (ii) $\mathrm{TAG} \geq 110 \mathrm{mg} / \mathrm{dl}$; (iii) HDL-C $\leq 40 \mathrm{mg} / \mathrm{dl}$; (iv) either SBP or DBP $\geq 90$ th percentile for age and sex, use of blood pressure-lowering medication or a previous diagnosis of hypertension; and (v) FPG $\geq 100 \mathrm{mg} / \mathrm{dl}$, current diabetes status or current use of insulin or hypoglycaemic medication.

\section{Statistical analyses}

Statistical analysis was performed using the statistical software package SAS version $9 \cdot 2$ for Windows and two-sided $P$ values of $<0 \cdot 05$ were considered statistically significant. Concentrations of serum $25(\mathrm{OH}) \mathrm{D}$ according to demographic variables and anthropometric and haematological characteristics of the participants are presented as means with their standard errors. Correlation analysis was performed to assess the relationships between serum $25(\mathrm{OH}) \mathrm{D}$ concentration and cardiometabolic risk factors. Student's $t$ test or one-way ANOVA was used to investigate the differences in serum $25(\mathrm{OH}) \mathrm{D}$ concentration according to demographic variables. Student's $t$ test was applied to compare the means of cardiometabolic risk factors across the 25(OH)D level categories. We categorized 25(OH)D $\leq 50 \mathrm{nmol} / \mathrm{l}(20 \mathrm{ng} / \mathrm{ml})$ as insufficiency and $>50 \mathrm{nmol} / \mathrm{l}$ as sufficiency using a broader definition in children and adolescents ${ }^{(8,9)}$. We used univariate and hierarchical multivariate logistic regression analyses to assess the associations of serum $25(\mathrm{OH}) \mathrm{D}$ concentration with the prevalence of metabolic syndrome and its individual components, such as abdominal obesity, high blood pressure, hyperglycaemia, hypertriacylglycerolaemia, low HDL-C, and overweight or obesity. Odds ratios and 95\% confidence intervals were estimated after adjustment for potential confounders. In multivariate analyses of the prevalence of metabolic syndrome, abdominal obesity, overweight or obesity, we first adjusted for age and gender (Model 1). We then we adjusted for the same variables as Model 1 plus regular physical exercise and alcohol drinking (Model 2). In addition, we adjusted for the variables in Model 2 plus multivitamin or mineral supplements use (Model 3). In multivariate analyses of the prevalence of high blood pressure, hyperglycaemia, low HDL-C and hypertriacylglycerolaemia as components of metabolic syndrome, we first adjusted for age and gender (Model 1). We then adjusted for the same variables as Model 1 plus BMI (Model 1a). In addition, we adjusted for the variables in Model 1a plus regular physical exercise and alcohol drinking (Model 2a). Finally, we adjusted for the variables in Model 2a plus multivitamin or mineral supplements use (Model 3a).

\section{Results}

\section{Baseline characteristics of the study population}

There were 1504 adolescents ( 792 boys and 712 girls) in the present study. Mean serum $25(\mathrm{OH}) \mathrm{D}$ concentration was $41 \cdot 7(\operatorname{se~} 0 \cdot 6) \mathrm{nmol} / \mathrm{l}(43 \cdot 2(\mathrm{se} 0 \cdot 8) \mathrm{nmol} / \mathrm{l}$ in boys and $40 \cdot 7(\operatorname{se} 0 \cdot 8) \mathrm{nmol} / \mathrm{l}$ in girls). We found that $75.3 \%$ of adolescents had $25(\mathrm{OH}) \mathrm{D}$ concentration below $50 \mathrm{nmol} / \mathrm{l}$ $(20 \mathrm{ng} / \mathrm{ml})$, and $10 \cdot 8 \%$ had $25(\mathrm{OH}) \mathrm{D}$ concentration below $25 \mathrm{nmol} / \mathrm{l}$ (11 ng/ml; Fig. 1). Table 1 displays mean concentrations of serum $25(\mathrm{OH}) \mathrm{D}$ according to the demographic characteristics of the participants. Serum $25(\mathrm{OH}) \mathrm{D}$ concentration was significantly higher in boys 
than in girls $(P<0 \cdot 001)$, in the $12-15$-year-olds than in the 16-18-year-olds $(P<0 \cdot 001)$, in those who drink alcohol at least once monthly than in those who drink less than once monthly $(P=0.003)$ and in those who exercise regularly than in those who do not exercise regularly $(P=0 \cdot 001)$. Monthly income, smoking status, multivitamin or mineral

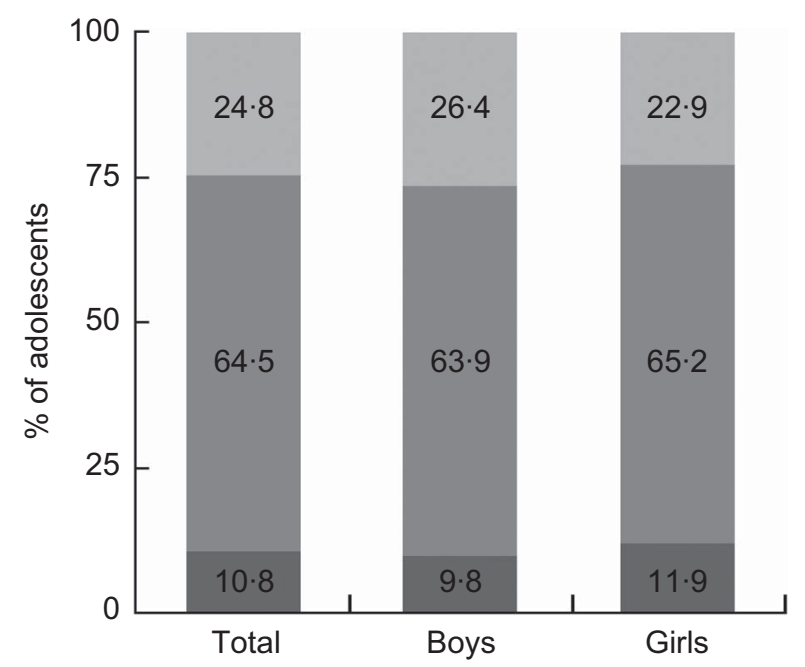

Fig. 1 Distribution of serum 25-hydroxyvitamin $\mathrm{D}(25(\mathrm{OH}) \mathrm{D})$ concentration $(\square, 25(\mathrm{OH}) \mathrm{D}<25 \mathrm{nmol} / \mathrm{l} ; \quad, \quad 25 \leq 25(\mathrm{OH}) \mathrm{D}$ $\leq 50 \mathrm{nmol} / \mathrm{l} ; \quad, \quad 25(\mathrm{OH}) \mathrm{D}>50 \mathrm{nmol} / \mathrm{l})$ by sex among Korean adolescents ( $n$ 1504) aged $12-18$ years, Korea National Health and Nutrition Examination Survey (KNHANES) 2008-2009 supplements use and sleep duration did not correlate with serum 25(OH)D concentration. There were no differences between those with insufficient and sufficient serum $25(\mathrm{OH}) \mathrm{D}$ concentration in terms of daily energy or $\mathrm{Ca}$ intake $(P=0.667$ for energy intake and $P=0.734$ for Ca intake).

\section{Associations between serum 25-bydroxyvitamin D concentration and cardiometabolic risk factors}

Table 2 shows the correlations of serum 25(OH)D concentration with cardiometabolic risk factors. There was a significant negative correlation of serum 25(OH)D with BMI, WC, SBP and DBP ( $r=-0 \cdot 080, P=0 \cdot 002$ for BMI; $r=-0 \cdot 071, P=0 \cdot 006$ for WC; $r=-0 \cdot 059, P=0 \cdot 021$ for SBP; $r=-0 \cdot 141, P<0 \cdot 001$ for DBP). Table 3 shows mean levels of cardiometabolic risk factors according to serum 25(OH)D concentration. BMI, WC and DBP showed significant differences between the insufficient and sufficient serum 25(OH)D groups. The $P$ values were $<0 \cdot 001$ for BMI, $<0 \cdot 001$ for WC and $0 \cdot 002$ for DBP.

\section{Prevalence of metabolic syndrome according to serum 25-bydroxyvitamin D level}

Table 4 shows the number of adolescents with each component of the metabolic syndrome. Table 5 presents the results of the multivariate logistic regression analyses to assess the association between 25(OH)D status and prevalence of metabolic syndrome. The proportion of

Table 1 Mean serum 25(OH)D concentration (nmol/l) according to demographic characteristics among Korean adolescents $(n 1504)$ aged 12-18 years, KNHANES 2008-2009

\begin{tabular}{|c|c|c|c|c|}
\hline & Unweighted $n$ & Mean & SE & $P$ value* \\
\hline Gender & & & & $<0.001$ \\
\hline Boys & 792 & $43 \cdot 2$ & $0 \cdot 8$ & \\
\hline Girls & 712 & $40 \cdot 7$ & $0 \cdot 8$ & \\
\hline Age (years) & & & & $<0.001$ \\
\hline $12-15$ & 959 & $44 \cdot 1$ & 0.8 & \\
\hline $16-18$ & 545 & $39 \cdot 1$ & $0 \cdot 7$ & \\
\hline Monthly income $(€)$ & & & & 0.801 \\
\hline$<712$ & 126 & $41 \cdot 1$ & $1 \cdot 6$ & \\
\hline $712-2135$ & 540 & $42 \cdot 1$ & 0.9 & \\
\hline$>2135$ & 820 & $42 \cdot 3$ & $0 \cdot 8$ & \\
\hline Alcohol drinking & & & & 0.003 \\
\hline Yes & 150 & $38 \cdot 7$ & $1 \cdot 3$ & \\
\hline No & 1351 & $42 \cdot 5$ & 0.7 & \\
\hline Smoking status & & & & 0.410 \\
\hline Yes & 110 & $40 \cdot 8$ & 0.7 & \\
\hline No & 1394 & $42 \cdot 2$ & $1 \cdot 6$ & \\
\hline Multivitamin or mineral supplement use & & & & $0 \cdot 228$ \\
\hline Yes & 151 & $43 \cdot 7$ & $1 \cdot 6$ & \\
\hline No & 1353 & $41 \cdot 9$ & $0 \cdot 7$ & \\
\hline Regular physical exercise & & & & 0.001 \\
\hline Yes & 458 & $44 \cdot 1$ & $1 \cdot 0$ & \\
\hline No & 1040 & $41 \cdot 1$ & $0 \cdot 7$ & \\
\hline Sleep duration (h) & & & & $0 \cdot 206$ \\
\hline$<6$ & 164 & $40 \cdot 6$ & 1.5 & \\
\hline $6-8$ & 1070 & $41 \cdot 9$ & 0.6 & \\
\hline$>8$ & 265 & $43 \cdot 6$ & $1 \cdot 3$ & \\
\hline
\end{tabular}

25(OH)D, 25-hydroxyvitamin D; KNHANES, Korea National Health and Nutrition Examination Survey.

${ }^{\star} P$ values were obtained by Student's $t$ test or one-way ANOVA. 
Table 2 Correlation of serum 25(OH)D concentrations with cardiometabolic risk factors among Korean adolescents ( $n$ 1504) aged 12-18 years, KNHANES 2008-2009

\begin{tabular}{lcc}
\hline & $r$ & $P$ value \\
\hline BMI $\left(\mathrm{kg} / \mathrm{m}^{2}\right)$ & -0.080 & 0.002 \\
WC $(\mathrm{cm})$ & -0.071 & 0.006 \\
SBP $(\mathrm{mmHg})$ & -0.059 & 0.021 \\
DBP $(\mathrm{mmHg})$ & -0.141 & $<0.0001$ \\
FPG (mg/dl) & 0.006 & 0.820 \\
HOMA-IR & -0.035 & 0.176 \\
TC (mg/dl) & 0.037 & 0.150 \\
HDL-C (mg/dl) & -0.046 & 0.073 \\
TAG (mg/dl) & -0.0003 & $0.990+$ \\
WBC $\left(\times 10^{9} / \mathrm{l}\right)$ & 0.019 & 0.470 \\
\hline
\end{tabular}

25(OH)D, 25-hydroxyvitamin D; KNHANES, Korea National Health and Nutrition Examination Survey; $r$, correlation coefficient; WC, waist circumference; SBP, systolic blood pressure; DBP, diastolic blood pressure; FPG, fasting plasma glucose; HOMA-IR, homeostasis model assessment of insulin resistance; TC, total cholesterol; HDL-C, HDL-cholesterol; WBC, white blood cell.

${ }^{*} P$ values were obtained by Pearson's correlation analyses.

tLog-transformation of TAG values was performed to calculate $r$ and $P$ value.

Table 3 Mean levels of cardiometabolic characteristics by serum $25(\mathrm{OH}) \mathrm{D}$ concentration among Korean adolescents $(n$ 1504) aged 12-18 years, KNHANES 2008-2009

\begin{tabular}{|c|c|c|c|c|c|}
\hline & \multicolumn{4}{|c|}{ Serum $25(\mathrm{OH}) \mathrm{D}$ concentration $(\mathrm{nmol} / \mathrm{l})$} & \multirow[b]{3}{*}{$P$ value* } \\
\hline & \multicolumn{2}{|c|}{$\leq 50$} & \multicolumn{2}{|c|}{$>50$} & \\
\hline & Mean & $\mathrm{SE}$ & Mean & SE & \\
\hline BMI $\left(\mathrm{kg} / \mathrm{m}^{2}\right)$ & $21 \cdot 2$ & $0 \cdot 1$ & $20 \cdot 4$ & 0.2 & $<0.0001$ \\
\hline$W C(\mathrm{~cm})$ & $71 \cdot 4$ & 0.4 & $69 \cdot 0$ & 0.5 & $<0.0001$ \\
\hline $\mathrm{SBP}(\mathrm{mmHg})$ & $107 \cdot 1$ & 0.4 & $105 \cdot 8$ & 0.7 & 0.201 \\
\hline $\mathrm{DBP}(\mathrm{mmHg})$ & $68 \cdot 6$ & 0.4 & $66 \cdot 4$ & 0.5 & 0.002 \\
\hline FPG $(\mathrm{mg} / \mathrm{dl})$ & $88 \cdot 8$ & 0.3 & $89 \cdot 3$ & 0.4 & 0.377 \\
\hline HOMA-IR & $2 \cdot 9$ & $0 \cdot 1$ & $2 \cdot 7$ & $0 \cdot 1$ & 0.062 \\
\hline TC (mg/dl) & $154 \cdot 7$ & $1 \cdot 1$ & $158 \cdot 9$ & $1 \cdot 7$ & 0.068 \\
\hline HDL-C (mg/dl) & $53 \cdot 4$ & 0.4 & $53 \cdot 1$ & 0.6 & 0.457 \\
\hline TAG (mg/dl) & $88 \cdot 3$ & $2 \cdot 4$ & $87 \cdot 0$ & $2 \cdot 8$ & $0.788+$ \\
\hline WBC $\left(\times 10^{9} / I\right)$ & $6 \cdot 2$ & $0 \cdot 1$ & $6 \cdot 2$ & $0 \cdot 1$ & 0.293 \\
\hline
\end{tabular}

25(OH)D, 25-hydroxyvitamin D; KNHANES, Korea National Health and Nutrition Examination Survey; WC, waist circumference; SBP, systolic blood pressure; DBP, diastolic blood pressure; FPG, fasting plasma glucose; HOMA-IR, homeostasis model assessment of insulin resistance; TC, total cholesterol; HDL-C, HCL-cholesterol; WBC, white blood cell.

${ }^{*} P$ values were obtained by Student's $t$ test.

tLog-transformation of TAG values was performed to calculate $P$ value. adolescents with metabolic syndrome in the insufficient serum $25(\mathrm{OH}) \mathrm{D}$ group was higher than that in the sufficient serum $25(\mathrm{OH}) \mathrm{D}$ group $(P=0 \cdot 029)$. Serum $25(\mathrm{OH}) \mathrm{D}$ concentration was significantly associated with the prevalence of metabolic syndrome in unadjusted $(P=0.038)$ and multivariate-adjusted logistic regression analyses after adjusting for age and gender $(P=0 \cdot 049$; Model 1$)$. Also, this correlation remained after adjusting for age, gender, regular physical exercise and alcohol drinking $(P=0.043$; Model 2). The likelihood of adolescents having metabolic syndrome in the insufficient serum 25(OH)D group was $2 \cdot 04$ (95\% CI 1.00, $4 \cdot 15 ; P=0 \cdot 049)$ compared with those in the sufficient group in Model 1 and 2.10 (95\% CI 1·02, 4·30; $P=0.043)$ in Model 2. After additional adjustment for use of multivitamin or mineral supplements, there was borderline statistical significance $(P=0 \cdot 053$; Model 3$)$.

\section{Risk for components of metabolic syndrome according to serum 25-bydroxyvitamin $D$ concentration}

Odds ratios for serum $25(\mathrm{OH}) \mathrm{D}$ concentration in relation to the prevalence of components of metabolic syndrome are presented in Table 6. Adolescents in the insufficient $25(\mathrm{OH}) \mathrm{D}$ group had an OR of $2 \cdot 13$ for abdominal obesity compared with the sufficient group after adjustment for age and gender (95\% CI 1·23, 3.67; P=0.007; Model 1). This relationship was unchanged after further adjustment for regular physical exercise and alcohol drinking $(\mathrm{OR}=2 \cdot 10$; $95 \%$ CI $1.24,3.55 ; P=0 \cdot 006 ;$ Model 2). The association remained after additionally controlling for supplement use (OR $=2 \cdot 05$; $95 \%$ CI 1·20, 3.49; $P=0 \cdot 008$; Model 3). Risks for high blood pressure, hyperglycaemia, low HDL-C level and hypertriacylglycerolaemia were not significantly associated with serum 25(OH)D status after adjustment for age, gender and BMI (Model 1a). These associations were unchanged after additional adjustment for regular physical exercise and alcohol drinking (Model 2a). These associations also persisted after adjustment for supplement use (Model 3a). We also conducted an analysis of the association between serum 25(OH)D concentration and BMI. Risk for overweight or obesity was significantly greater in the

Table 4 Distribution of abnormalities of each component of metabolic syndrome by age group among Korean adolescents ( $n$ 1504) aged 12-18 years, KNHANES 2008-2009

\begin{tabular}{|c|c|c|c|c|c|}
\hline & \multicolumn{2}{|c|}{$12-15$ years } & \multicolumn{2}{|c|}{$16-18$ years } & \multirow[b]{2}{*}{ Total $n$} \\
\hline & $n$ & $\%$ & $n$ & $\%$ & \\
\hline Metabolic syndrome & 33 & $3 \cdot 6$ & 31 & $4 \cdot 6$ & 64 \\
\hline \multicolumn{6}{|c|}{ Components of metabolic syndrome } \\
\hline Abdominal obesity & 62 & $5 \cdot 8$ & 56 & $9 \cdot 1$ & 118 \\
\hline High blood pressure & 265 & $28 \cdot 8$ & 169 & $31 \cdot 6$ & 434 \\
\hline Hyperglycaemia & 56 & $5 \cdot 8$ & 20 & $4 \cdot 5$ & 76 \\
\hline Low HDL-C & 91 & $9 \cdot 9$ & 63 & $9 \cdot 7$ & 154 \\
\hline Hypertriacylqlycerolaemia & 226 & $23 \cdot 4$ & 119 & $20 \cdot 7$ & 345 \\
\hline Overweight or obesity & 166 & $17 \cdot 7$ & 104 & $18 \cdot 9$ & 270 \\
\hline
\end{tabular}

KNHANES, Korea National Health and Nutrition Examination Survey; HDL-C, HDL-cholesterol. 
Table 5 Multivariate-adjusted odds ratios and $95 \%$ confidence intervals for prevalence of metabolic syndrome according to serum 25(OH)D concentration among Korean adolescents $(n$ 1504) aged $12-18$ years, KNHANES 2008-2009

\begin{tabular}{|c|c|c|c|c|c|}
\hline & \multicolumn{4}{|c|}{ Serum $25(\mathrm{OH}) \mathrm{D}$ concentration $(\mathrm{nmol} / \mathrm{l})$} & \multirow[b]{3}{*}{$P$ value } \\
\hline & \multicolumn{2}{|c|}{$\leq 50$} & \multicolumn{2}{|c|}{$>50$} & \\
\hline & OR & $95 \% \mathrm{Cl}$ & OR & $95 \% \mathrm{Cl}$ & \\
\hline Prevalence, $n(\%)$ & 54 & $4 \cdot 6$ & 10 & $2 \cdot 2$ & $0.029 \S$ \\
\hline Unadjusted & $2 \cdot 16$ & $1 \cdot 04,4 \cdot 46$ & $1 \cdot 00$ & Ref. & $0.038 \|$ \\
\hline Model $1^{*}$ & $2 \cdot 04$ & $1 \cdot 00,4 \cdot 15$ & 1.00 & Ref. & 0.049 \\
\hline Model $2 \dagger$ & $2 \cdot 10$ & $1 \cdot 02,4 \cdot 30$ & $1 \cdot 00$ & Ref. & $0.043^{\circ}$ \\
\hline Model 3‡ & $2 \cdot 04$ & $0 \cdot 99,4 \cdot 18$ & $1 \cdot 00$ & Ref. & 0.053 \\
\hline
\end{tabular}

25(OH)D, 25-hydroxyvitamin D; KNHANES, Korea National Health and Nutrition Examination Survey; Ref., referent category.

${ }^{*}$ Adjusted for age and gender.

†Adjusted for variables in Model 1 plus regular physical exercise and alcohol drinking.

$\ddagger$ Adjusted for variables in Model 2 plus use of multivitamin or mineral supplements.

$\S P$ value was obtained by the $\chi^{2}$ test.

$\| P$ value was obtained by unadjusted logistic regression analysis.

- $P$ values were obtained by multivariate-adjusted logistic regression analyses.

Table 6 Multivariate-adjusted odds ratios and $95 \%$ confidence intervals for cardiometabolic risk factors according to serum 25(OH)D concentration among Korean adolescents $(n$ 1504) aged 12-18 years, KNHANES 2008-2009

\begin{tabular}{|c|c|c|c|}
\hline \multirow[b]{2}{*}{ Components of metabolic syndrome } & \multicolumn{2}{|c|}{ Serum $25(\mathrm{OH}) \mathrm{D} \leq 50 \mathrm{nmol} / \mathrm{l}$} & \multirow[b]{2}{*}{$P$ value } \\
\hline & OR & $95 \% \mathrm{Cl}$ & \\
\hline \multicolumn{4}{|l|}{ Abdominal obesity } \\
\hline Model 1t & $2 \cdot 13$ & $1.23,3.67$ & 0.007 \\
\hline Model $2 \ddagger$ & $2 \cdot 10$ & $1 \cdot 24,3 \cdot 55$ & 0.006 \\
\hline Model $3 \S$ & $2 \cdot 05$ & $1 \cdot 20,3 \cdot 49$ & 0.008 \\
\hline \multicolumn{4}{|l|}{ High blood pressure } \\
\hline Model $1 \dagger$ & $1 \cdot 16$ & $0.84,1.60$ & $0 \cdot 367$ \\
\hline Model 1all & $1 \cdot 11$ & $0.80,1.54$ & 0.525 \\
\hline Model 2a & $1 \cdot 08$ & $0.77,1.50$ & 0.660 \\
\hline Model $3 a^{\star \star}$ & $1 \cdot 07$ & $0.77,1.50$ & 0.673 \\
\hline \multicolumn{4}{|l|}{ Hyperglycaemia } \\
\hline Model 1t & 0.98 & $0.54,1 \cdot 76$ & 0.933 \\
\hline Model 1all & 0.89 & $0.49,1.62$ & $0 \cdot 696$ \\
\hline Model 2a & 0.95 & $0.53,1.72$ & $0 \cdot 866$ \\
\hline Model $3 a^{\star \star}$ & 0.95 & $0 \cdot 52,1 \cdot 72$ & 0.865 \\
\hline \multicolumn{4}{|l|}{ Low HDL-C } \\
\hline Model 1† & $1 \cdot 07$ & $0.70,1 \cdot 66$ & $0 \cdot 750$ \\
\hline Model 1all & 0.94 & $0 \cdot 60,1 \cdot 46$ & $0 \cdot 774$ \\
\hline Model 2a & 0.85 & $0.54,1.34$ & $0 \cdot 485$ \\
\hline Model $3 a^{\star \star}$ & 0.85 & $0.54,1.34$ & $0 \cdot 474$ \\
\hline \multicolumn{4}{|l|}{ Hypertriacylglycerolaemia } \\
\hline Model $1 \dagger$ & $1 \cdot 08$ & $0 \cdot 78,1 \cdot 48$ & 0.648 \\
\hline Model 1all & 0.96 & $0 \cdot 70,1 \cdot 32$ & $0 \cdot 807$ \\
\hline Model 2a & 0.93 & $0.67,1.23$ & 0.655 \\
\hline Model $3 a^{\star \star}$ & 0.93 & $0 \cdot 68,1 \cdot 29$ & $0 \cdot 671$ \\
\hline \multicolumn{4}{|l|}{ Overweight or obesity } \\
\hline Model 1† & 1.52 & $1 \cdot 04,2 \cdot 22$ & 0.030 \\
\hline Model 2ł & $1 \cdot 51$ & $1 \cdot 05,2 \cdot 19$ & 0.028 \\
\hline Model $3 \S$ & $1 \cdot 49$ & $1 \cdot 03,2 \cdot 16$ & 0.035 \\
\hline
\end{tabular}

25(OH)D, 25-hydroxyvitamin D; KNHANES, Korea National Health and Nutrition Examination Survey; HDL-C, HDL-cholesterol.

Referent category was serum $25(\mathrm{OH}) \mathrm{D}>50 \mathrm{nmol} / \mathrm{l}$.

${ }^{*} P$ values were obtained by multivariate-adjusted logistic regression analyses.

tAdjusted for age and gender.

‡Adjusted for variables in Model 1 plus regular physical exercise and alcohol drinking.

\$Adjusted for variables in Model 2 plus use of multivitamin or mineral supplement.

॥Adjusted for variables in Model 1 plus BMI.

$\uparrow$ Adjusted for variables in Model 1 a plus regular physical exercise and alcohol drinking.

${ }^{\star *}$ Adjusted for variables in Model 2a plus use of multivitamin or mineral supplements.

insufficient serum 25(OH)D group compared with the sufficient group, even after adjustment for confounders (OR $=1.52 ; 95 \%$ CI $1.04,2 \cdot 22 ; P=0.030$ in Model 1 ; OR $=1.51 ; 95 \%$ CI $1.05,2 \cdot 19 ; P=0.028$ in Model 2 ; $\mathrm{OR}=1 \cdot 49 ; 95 \% \mathrm{CI} 1 \cdot 03,2 \cdot 16 ; P=0 \cdot 035$ in Model 3 ).

\section{Discussion}

Metabolic syndrome is known as the most serious risk factor for type 2 diabetes mellitus, as well as for $\mathrm{CVD}^{(21)}$. It is a cluster of atherosclerotic CVD risk factors, including 
central obesity, dyslipidaemia, insulin resistance and hypertension, and non-traditional components such as non-alcoholic fatty liver disease and systemic chronic inflammation $^{(22,23)}$. The pathophysiology of metabolic syndrome has not been accurately established; however, insulin resistance and obesity are considered to be among the significant mechanisms involved ${ }^{(24)}$.

Although the mechanisms for improvement of insulin sensitivity by vitamin $\mathrm{D}$ in the prevention of CVD have not been proven, some plausible hypotheses exist ${ }^{(25,26)}$. It has been suggested that the immune-modulating action of vitamin $\mathrm{D}$ through $\mathrm{T}$ helper cells protects pancreatic $\beta$-cells from chronic inflammation and increases Ca levels in the $\beta$-cells to increase participation in insulin secretion via the up-regulation of insulin receptors ${ }^{(25)}$. Another mechanism includes increases in intracellular $\mathrm{Ca}$, necessary for insulin release from proinsulin ${ }^{(26)}$. Vitamin D is thought to inhibit the renin-angiotensin system to restrain blood pressure increases and to control T helper cells to inhibit inflammation, atherosclerosis, thrombosis and vascular calcification, which prevents the development of $\mathrm{CVD}^{(27,28)}$, and overactivity in the renin-angiotensin system in islets has been reported to be reduced by activated vitamin $\mathrm{D}^{(29)}$. Vitamin $\mathrm{D}$ is also known to inhibit the cell cycle in the heart and to restrict mechanisms causing myocardial fibrosis, improving myocardial structure or contractility ${ }^{(30)}$.

Obesity, another important element of metabolic syndrome, is reported to be associated with vitamin D deficiency; however, the relevant mechanism is not explicitly known. Recent research supports the idea that sequestration of vitamin $\mathrm{D}$ in fat is a central problem in obesity $^{(31)}$. Although there is no difference in synthesis of vitamin D irrespective of obesity, highly lipid-soluble $25(\mathrm{OH}) \mathrm{D}$ is stored in fat, decreasing bioavailability ${ }^{(32)}$. Overweight or obese people are less likely to be exposed to UV light due to infrequent outdoor physical activity, thus increasing the risk of a low serum vitamin D level ${ }^{(4)}$.

Multiple prior studies have investigated the relevance of serum 25(OH)D concentration to metabolic syndrome and cardiometabolic risk factors without consistent results. Many cross-sectional and a few prospective studies have found reverse correlations between serum $25(\mathrm{OH}) \mathrm{D}$ concentration and metabolic syndrome, glucose intolerance, insulin resistance and type 2 diabetes mellitus ${ }^{(10,33-35)}$. In a randomized controlled trial in Asians, insulin resistance was reduced when serum $25(\mathrm{OH}) \mathrm{D}$ concentration was raised to $80 \mathrm{nmol} / \mathrm{l}(>32 \mathrm{ng} / \mathrm{ml})$ or more ${ }^{(36)}$. Vitamin $\mathrm{D}$ has been reported to improve endothelial function and insulin resistance in diabetic patients ${ }^{(37)}$. Serum $25(\mathrm{OH}) \mathrm{D}$ has been reported to be associated with the risk for CVD, including hypertension, IHD, heart failure and mortality from these diseases $^{(11,13,38-40)}$. In contrast, some population-based studies have not shown an independent contribution by serum 25(OH)D concentration in metabolic syndrome ${ }^{(41,42)}$, and other small-scale intervention studies have found that vitamin D supplementation has no effect on insulin resistance or sensitivity ${ }^{(43-45)}$. Furthermore, several studies have not agreed on a consistent relevance between components of metabolic syndrome and serum $25(\mathrm{OH}) \mathrm{D}$ concentration $^{(46)}$. In particular, the relevance of vitamin D status for metabolic syndrome risk among non-Western populations has not yet been elucidated ${ }^{(10)}$. Study efforts have not focused on elucidating the relationship between vitamin D status and cardiometabolic risk factors among children and adolescents as a specific group.

We have demonstrated that vitamin D insufficiency is common in Korean adolescents. Based on our study, $75.3 \%$ of Korean adolescents had vitamin D insufficiency or deficiency and a mean concentration of serum $25(\mathrm{OH}) \mathrm{D}$ that is much lower than that of US adolescents in the National Health and Nutrition Examination Survey 2001-2004 ${ }^{(45)}$, while $18.0 \%$ of them were overweight or obese. Mean concentration of serum 25(OH)D was significantly different according to age, gender, regular physical exercise and alcohol drinking and we regarded these factors as determinants of vitamin D status. We measured well-known cardiometabolic risk factors, such as BMI and DBP, and these factors varied with vitamin D status. Vitamin D insufficiency was associated with an increased risk of the prevalence of metabolic syndrome in Korean adolescents. WC and BMI were the most strongly correlated cardiometabolic components in relation to serum 25(OH)D status. Our study did not identify any differences by serum $25(\mathrm{OH}) \mathrm{D}$ group for indicators of insulin resistance.

The present study is the first one on the associations between serum $25(\mathrm{OH}) \mathrm{D}$ concentration and cardiometabolic risk factors in Korean adolescents and has considerable significance in that it used nationally representative data. However, our study has some limitations. As a cross-sectional and retrospective study, the associations it demonstrated do not imply a causal relationship among serum $25(\mathrm{OH}) \mathrm{D}$ concentration, metabolic syndrome and cardiometabolic risk factors. The study may be confounded by a lack of data on relevant variables such as information on total dietary vitamin D intake, season of measurement, sunshine exposure and factors that affect vitamin D synthesis such as clothing and sunscreen use, and Tanner stages of sexual development. In addition, the assessment of vitamin $\mathrm{D}$ status was by only a single measured value and serum 25(OH)D levels measured in January, which are expected to be the lowest of the year, were not included in the study.

The pathological precursors of CVD seem to develop during childhood ${ }^{(47)}$. Metabolic syndrome in childhood and adolescence is likely to increase the risk of the prevalence of CVD and type 2 diabetes mellitus during adulthood $^{(48)}$. Hence, it is important to detect metabolic syndrome in adolescence so that attempts can be made to prevent later sequelae. Accordingly, much more attention should be paid to the associations among vitamin D 
repletion and metabolic syndrome and CVD. Basic research on relevant mechanisms is needed with interventional trial data to determine if oral supplementation with vitamin D can improve cardiovascular outcomes. At the same time, race- and age-specific optimal serum concentrations of $25(\mathrm{OH}) \mathrm{D}$ and recommendations for vitamin D intake should be developed allowing for the potentially multifarious effects of adequate vitamin D repletion on human health.

\section{Acknowledgements}

Sources of funding: This research received no specific grant from any funding agency in the public, commercial or not-for-profit sector. Conflicts of interest: All authors declare that they have no financial or other substantive conflict of interest that might be construed to influence the results or interpretation of the manuscript. Authors contributions: G.E.N. and D.H.K. contributed to conception and design of the study, collected and analysed the data, interpreted the results and wrote the article. K.H.C. contributed to conception and design of the study, wrote the article and supervised the work. Y.G.P. and K.D.H. collected and analysed the data, interpreted the results and wrote the article. S.M.K., S.H.L., B.J.K. and M.J.K. were involved in conception and design of the study and wrote the article. G.E.N. and D.H.K. contributed to this work equally. Acknowledgement: The authors thank the participants in the 2008-2009 KNHANES.

\section{References}

1. Parfitt AM, Gallagher JC, Heaney RP et al. (1982) Vitamin D and bone health in the elderly. Am J Clin Nutr 36, 1014-1031.

2. Holick MF (2007) Vitamin D deficiency. N Engl J Med 357, 266-281.

3. Cashman KD (2007) Vitamin D in childhood and adolescence. Postgrad Med J 83, 230-235.

4. Tsiaras WG \& Weinstock MA (2011) Factors influencing vitamin D status. Acta Derm Venereol 91, 115-124.

5. Reis JP, von Muhlen D, Miller ER 3rd et al. (2009) Vitamin D status and cardiometabolic risk factors in the United States adolescent population. Pediatrics 124, e371-e379.

6. Donin AS, Nightingale CM, Owen CG et al. (2010) Nutritional composition of the diets of South Asian, black African-Caribbean and white European children in the United Kingdom: the Child Heart and Health Study in England (CHASE). Br J Nutr 104, 276-285.

7. Muller DN, Kleinewietfeld M \& Kvakan H (2011) Vitamin D review. J Renin Angiotensin Aldosterone Syst 12, 125-128.

8. Misra M, Pacaud D, Petryk A et al. (2008) Vitamin D deficiency in children and its management: review of current knowledge and recommendations. Pediatrics 122, 398-417.

9. Gordon CM, DePeter KC, Feldman HA et al. (2004) Prevalence of vitamin D deficiency among healthy adolescents. Arch Pediatr Adolesc Med 158, 531-537.

10. Kim MK, Il Kang M, Won Oh K et al. (2010) The association of serum vitamin $\mathrm{D}$ level with presence of metabolic syndrome and hypertension in middle-aged Korean subjects. Clin Endocrinol (Oxf) 73, 330-338.
11. Judd SE \& Tangpricha V (2009) Vitamin D deficiency and risk for cardiovascular disease. Am J Med Sci 338, 40-44.

12. Forouhi NG, Luan J, Cooper A et al. (2008) Baseline serum 25-hydroxy vitamin $\mathrm{D}$ is predictive of future glycemic status and insulin resistance: the Medical Research Council Ely Prospective Study 1990-2000. Diabetes 57, 2619-2625.

13. Dobnig H, Pilz S, Scharnagl H et al. (2008) Independent association of low serum 25-hydroxyvitamin $\mathrm{D}$ and 1,25-dihydroxyvitamin D levels with all-cause and cardiovascular mortality. Arch Intern Med 168, 1340-1349.

14. Lu L, Yu Z, Pan A et al. (2009) Plasma 25-hydroxyvitamin D concentration and metabolic syndrome among middleaged and elderly Chinese individuals. Diabetes Care 32, 1278-1283.

15. Choi HS, Oh HJ, Choi $\mathrm{H}$ et al. (2011) Vitamin D insufficiency in Korea - a greater threat to younger generation: the Korea National Health and Nutrition Examination Survey (KNHANES) 2008. J Clin Endocrinol Metab 96, 643-651.

16. Choi HS, Kim KA, Lim CY et al. (2011) Low serum vitamin $\mathrm{D}$ is associated with high risk of diabetes in Korean adults. J Nutr 141, 1524-1528.

17. Korea Centers for Disease Control and Prevention (2008) Korea National Health and Nutrition Examination Survey (KNHANES). http://knhanes.cdc.go.kr/ (accessed January 2011).

18. Expert Panel on Detection, Evaluation, and Treatment of High Blood Cholesterol in Adults (2001) Executive Summary of The Third Report of The National Cholesterol Education Program (NCEP) Expert Panel on Detection, Evaluation, and Treatment of High Blood Cholesterol in Adults (Adult Treatment Panel III). JAMA 285, 2486-2497.

19. Moon JS, Lee SY, Nam CM et al. (2008) 2007 Korean National Growth Charts: review of developmental process and an outlook. Korean J Pediatr 51, 1-25.

20. Wallace TM, Levy JC \& Matthews DR (2004) Use and abuse of HOMA modeling. Diabetes Care 27, 1487-1495.

21. Wilson PW, D'Agostino RB, Parise H et al. (2005) Metabolic syndrome as a precursor of cardiovascular disease and type 2 diabetes mellitus. Circulation 112, 3066-3072.

22. Angulo P \& Lindor KD (2002) Non-alcoholic fatty liver disease. J Gastroenterol Hepatol 17, Suppl., S186-S190.

23. Shoelson SE, Lee J \& Goldfine AB (2006) Inflammation and insulin resistance. J Clin Invest 116, 1793-1801.

24. Eckel RH, Grundy SM \& Zimmet PZ (2005) The metabolic syndrome. Lancet 365, 1415-1428.

25. Bikle D (2009) Nonclassic actions of vitamin D. J Clin Endocrinol Metab 94, 26-34.

26. Davidson HW, Rhodes CJ \& Hutton JC (1988) Intraorganellar calcium and $\mathrm{pH}$ control proinsulin cleavage in the pancreatic $\beta$ cell via two distinct site-specific endopeptidases. Nature 333, 93-96.

27. Verhave G \& Siegert CE (2010) Role of vitamin D in cardiovascular disease. Neth J Med 68, 113-118.

28. Mathew S, Lund RJ, Chaudhary LR et al. (2008) Vitamin D receptor activators can protect against vascular calcification. I Am Soc Nephrol 19, 1509-1519.

29. Cheng Q, Li YC, Boucher BJ et al. (2011) A novel role for vitamin D: modulation of expression and function of the local renin-angiotensin system in mouse pancreatic islets. Diabetologia 54, 2077-2081.

30. Artaza JN, Mehrotra R \& Norris KC (2009) Vitamin D and the cardiovascular system. Clin J Am Soc Nephrol 4, $1515-1522$.

31. Hypponen E \& Power C (2006) Vitamin D status and glucose homeostasis in the 1958 British birth cohort: the role of obesity. Diabetes Care 29, 2244-2246.

32. Wortsman J, Matsuoka LY, Chen TC et al. (2000) Decreased bioavailability of vitamin D in obesity. Am J Clin Nutr 72, 690-693. 
33. Pittas AG, Lau J, Hu FB et al. (2007) The role of vitamin D and calcium in type 2 diabetes. A systematic review and meta-analysis. J Clin Endocrinol Metab 92, 2017-2029.

34. Alemzadeh R, Kichler J, Babar G et al. (2008) Hypovitaminosis D in obese children and adolescents: relationship with adiposity, insulin sensitivity, ethnicity, and season. Metabolism 57, 183-191.

35. Ganji V, Zhang X, Shaikh N et al. (2011) Serum 25-hydroxyvitamin D concentrations are associated with prevalence of metabolic syndrome and various cardiometabolic risk factors in US children and adolescents based on assay-adjusted serum 25-hydroxyvitamin D data from NHANES 2001-2006. Am J Clin Nutr 94, 225-233.

36. von Hurst PR, Stonehouse W \& Coad J (2010) Vitamin D supplementation reduces insulin resistance in South Asian women living in New Zealand who are insulin resistant and vitamin D deficient - a randomised, placebo-controlled trial. Br J Nutr 103, 549-555.

37. Sugden JA, Davies JI, Witham MD et al. (2008) Vitamin D improves endothelial function in patients with type 2 diabetes mellitus and low vitamin D levels. Diabet Med 25, 320-325.

38. Forman JP, Giovannucci E, Holmes MD et al. (2007) Plasma 25-hydroxyvitamin D levels and risk of incident hypertension. Hypertension 49, 1063-1069.

39. Giovannucci E, Liu Y, Hollis BW et al. (2008) 25-Hydroxyvitamin D and risk of myocardial infarction in men: a prospective study. Arch Intern Med $\mathbf{1 6 8}$, 1174-1180.
40. Fiscella K \& Franks P (2010) Vitamin D, race, and cardiovascular mortality: findings from a national US sample. Ann Fam Med 8, 11-18.

41. Reis JP, von Muhlen D, Kritz-Silverstein D et al. (2007) Vitamin D, parathyroid hormone levels, and the prevalence of metabolic syndrome in community-dwelling older adults. Diabetes Care 30, 1549-1555.

42. Rueda S, Fernandez-Fernandez C, Romero F et al. (2008) Vitamin D, PTH, and the metabolic syndrome in severely obese subjects. Obes Surg 18, 151-154.

43. Fliser D, Stefanski A, Franek E et al. (1997) No effect of calcitriol on insulin-mediated glucose uptake in healthy subjects. Eur J Clin Invest 27, 629-633.

44. Pittas AG, Harris SS, Stark PC et al. (2007) The effects of calcium and vitamin D supplementation on blood glucose and markers of inflammation in nondiabetic adults. Diabetes Care 30, 980-986.

45. Pittas AG, Chung M, Trikalinos $\mathrm{T}$ et al. (2010) Systematic review: vitamin D and cardiometabolic outcomes. Ann Intern Med 152, 307-314.

46. Smotkin-Tangorra M, Purushothaman R, Gupta A et al. (2007) Prevalence of vitamin D insufficiency in obese children and adolescents. J Pediatr Endocrinol Metab 20, 817-823.

47. Berenson GS, Srinivasan SR, Bao W et al. (1998) Association between multiple cardiovascular risk factors and atherosclerosis in children and young adults. The Bogalusa Heart Study. N Engl J Med 338, 1650-1656.

48. Dietz WH (1998) Health consequences of obesity in youth: childhood predictors of adult disease. Pediatrics 101, 518-525. 